\title{
Application of Interpolation Inequalities to the Study of Operators with Linear Fractional Endpoint Singularities in Weighted Hölder Spaces
}

\author{
Oleksandr Karelin*, Anna Tarasenko \\ Institute of Basic Sciences and Engineering, Hidalgo State University, Pachuca, Mexico \\ Email: karelin@uaeh.edu.mx
}

Received 11 August 2014; revised 30 August 2014; accepted 8 September 2014

Copyright (C) 2014 by authors and Scientific Research Publishing Inc.

This work is licensed under the Creative Commons Attribution International License (CC BY). http://creativecommons.org/licenses/by/4.0/

\section{(c) (i) Open Access}

\begin{abstract}
In this paper we consider operators with endpoint singularities generated by linear fractional Carleman shift in weighted Hölder spaces. Such operators play an important role in the study of algebras generated by the operators of singular integration and multiplication by function. For the considered operators, we obtained more precise relations between norms of integral operators with local singularities in weighted Lebesgue spaces and norms in weighted Hölder spaces, making use of previously obtained general results. We prove the boundedness of operators with linear fractional singularities.
\end{abstract}

\section{Keywords}

Endpoint Singularities, Weighted Holder Space, Weighted Lebesgue Spaces, Relation between Norms, Boundedness

\section{Introduction}

The solvability theory of singular integral operators has developed independently in Hölder and Lebesgue spaces [1]-[7], as norms in these spaces differ widely in their structure.

The norm in weighted Hölder spaces is defined in the following way. A function $\varphi(x)$ that satisfies the following condition on contour $J=[0,1]$,

\footnotetext{
${ }^{*}$ Corresponding author.
}

How to cite this paper: Karelin, O. and Tarasenko, A. (2014) Application of Interpolation Inequalities to the Study of Operators with Linear Fractional Endpoint Singularities in Weighted Hölder Spaces. Applied Mathematics, 5, 2779-2885. 


$$
\left|\varphi\left(x_{1}\right)-\varphi\left(x_{2}\right)\right| \leq C\left|x_{1}-x_{2}\right|^{\mu}, \quad x_{1} \in J, \quad x_{2} \in J, \quad 0<\mu<1,
$$

is called Hölder function with exponent $\mu$ and constant $C$ on contour $J$.

Let $J$ be a power function which has zeros at the endpoints $x=0, x=1$ :

$$
h(x)=(x-0)^{\mu_{0}}(1-x)^{\mu_{1}}, \quad \mu<\mu_{0}<1+\mu, \quad \mu<\mu_{1}<1+\mu .
$$

The functions that become Hölder functions and turn into zero at the endpoints, after being multiplied by $h(x)$, form a Banach space of Hölder functions with weight $h$ :

$$
H_{\mu}^{0}(J, h), \quad J=[0,1] .
$$

The norm in space $H_{\mu}^{0}(J, h)$ is defined by

$$
\|f\|_{H_{\mu}^{0}(J, h)}=\|h f\|_{H_{\mu}(J)}
$$

where

$$
\|h f\|_{H_{\mu}(J)}=\|h f\|_{C}=\|h f\|_{\mu}
$$

and

$$
\begin{gathered}
\|h f\|_{C}=\max _{x \in J}|h(x) f(x)|, \\
\|h f\|_{\mu}=\sup _{x_{1}, x_{2} \in J, x_{1} \neq x_{2}}|h(x) f(x)|_{\mu},
\end{gathered}
$$

specifying that

$$
|h(x) f(x)|_{\mu}=\frac{\left|h\left(x_{1}\right) f\left(x_{1}\right)-h\left(x_{2}\right) f\left(x_{2}\right)\right|}{\left|x_{1}-x_{2}\right|^{\mu}} .
$$

We denote by $\Omega(X)$ the set of all bounded linear operators mapping the Banach space $X$ into $X$.

The norm of an operator $A \in \Omega$ will be denoted by $\|A\|_{\Omega(V)}$.

We denote a class of continuous functions on the segment $[0,1]$ by $C([0,1])$, also denote a class of differentiable functions on interval $(0,1)$ by $C^{1}((0,1))$, and we denote by $C^{01}((0,1), h)$ a class of functions

$$
\left\{\varphi \mid h_{\varphi} \in C^{1}((0,1)), \lim _{t \rightarrow j} h(t) \varphi(t)=0, j=0,1\right\}
$$

Let us introduce the following notation:

$$
(D v)(x)=x(1-x) \frac{\mathrm{d}}{\mathrm{d} x} v(x),
$$

$D^{0}$ is the identity operator $I, D^{1}=D, D^{n}=D^{n-1} D ; \chi_{x}(t)$ is the characteristic function of segment $[0, x], x \in(0,1)$.

Let $\rho$ be a power function which has zeros at the endpoints $x=0, x=1$ :

$$
\rho=(x-0)^{r}(1-x)^{s}, \quad-1<r<1-p, \quad-1<s<p-1, \quad p>1
$$

Let $L_{p}(J, \rho)$ denote the space of functions on $J$ which are integrable in the $p$-th power after multiplication by the weight-function $\rho$.

The norm in space $L_{p}(J, \rho)$ is defined by

$$
\|f\|_{L_{p}(J, \rho)}=\left(\iint_{J}|f(t)|^{p} \rho(t) \mathrm{d} t\right)^{\frac{1}{p}} .
$$


As we can see, the norms in spaces $H_{\mu}^{0}(J, h)$ and $L_{p}(J, \rho)$ are different in their character, and the presence of a direct connection should not be expected. However, in this work, we describe a class of operators with local singularities for which we were able to find inequalities that connect the norms in weighted Lebesgue spaces with the norms in weighted Hölder spaces. Operators with fixed singularities perform an essential role in the study of singular integral operators with shift [8]-[10], in particular in the construction of regularizations.

By way of representatives of such types of operators we may consider the following operators with local singularities:

$$
\begin{gathered}
\frac{1}{\pi} \int_{0}^{1} \frac{f(t) \mathrm{d} t}{t+x-2 t x}, \quad x \in(0,1), \\
\frac{1}{\pi} \int_{0}^{1} \frac{f(t) \mathrm{d} t}{t+k x}, \quad k>0, \quad x \in(0,1)
\end{gathered}
$$

Such operators can be used in the study of boundedness, of belonging of some operators to Banach algebras and of the solvability of operators in weighted Hölder spaces, on the basis of known results for operators in weighted Lebesgue spaces.

\section{Inequality Which Connects the Norms in Lebesque and Hölder Weighted Spaces}

It is useful to avoid two variables in the second term of the definition of the norm in Hölder spaces, for which we make use of

Lemma 1.

Let

$$
h(x) f(x) \in C([0,1]) \cap C^{1}((0,1))
$$

then

$$
\|h f\|_{H_{\mu}(J)} \leq C_{1}\left(\sup _{o<x<1} \frac{|h(x) f(x)|}{x^{\mu}(1-x)^{\mu}}+\sup _{o<x<1} \frac{|h(x) D f(x)|}{x^{\mu}(1-x)^{\mu}}\right),
$$

where $C_{1}$ is a constant which does not depend on $f(x)$.

On the basis of Lemma 1 the following theorem can be proved [11].

\section{Theorem 1.}

Let the following conditions hold for some operator $B$ :

1) Operators $D^{n} B ; n=0 ; 1 ; 2$ are bounded in spaces

$$
L_{q_{j}}\left(J, \rho_{j}\right), \quad 1<q_{j}<+\infty, \quad \rho_{j}(x)=x^{r_{j}}(1-x)^{s_{j}}, \quad-1<r_{j}<q_{j}-1, \quad-1<s_{j}<q_{j}-1, j=1,2 ;
$$

2) For any fixed $x \in(0 ; 1)$ and for any function $\varphi$ from space $H_{\mu}^{0}(J, h)$,

$$
h(x)=x^{\mu+r}(1-x)^{\mu+s} ; \quad 0<\mu<1 ; 0<r<1 ; 0<s<1
$$

the following properties are fulfilled:

$$
\begin{aligned}
& \left(D^{n} B \chi_{x} \varphi\right)(t) \in C^{01}((0,1), h), \\
& \left(D^{n} B\left(1-\chi_{x}\right) \varphi\right)(t) \in C^{01}((0,1), h), \quad n=0,1 .
\end{aligned}
$$

Moreover, inequalities

$$
\frac{1+r_{2}}{q_{2}}<r<\frac{1+r_{1}}{q_{1}}, \quad \frac{1+s_{1}}{q_{1}}<s<\frac{1+s_{2}}{q_{2}}
$$

are correct.

It follows that operator $B$ is bounded in space $H_{\mu}^{0}(J, h)$ and for its norm the following estimation is ful- 
filled

$$
\|B\|_{\Omega\left(H_{\mu}^{0}(J, h)\right)} \leq C_{2} \sum_{n=0}^{2}\left(\left\|D^{n} B\right\|_{\Omega\left(L_{L_{1}}\left(J, \rho_{1}\right)\right)}+\left\|D^{n} B\right\|_{\Omega\left(L_{q_{2}}\left(J, \rho_{2}\right)\right)}\right)
$$

where $C_{2}$ is a certain positive constant.

These results can be used in the study of operators in weighted Hölder spaces, on the basis of known results for operators in weighted Lebesgue spaces. In particular, operators with local endpoint singularities can be used in the construction of the left and the right regularizers in the study of Fredholmness of operators in weighted Hölder spaces.

\section{Operators with Linear Fractional Endpoint Singularities}

We formulate a useful assertion which follows directly from Theorem 1.

Corollary 1. Let properties (1) and (2) be correct for the operator $B=D_{1} R_{1}$ and furthermore

$$
\left\|D^{n} D_{1} R_{1}\right\|_{L_{q_{j}}\left(J, \rho_{j}\right)} \leq C_{3}\left\|D_{1} M R_{1}\right\|_{L_{q_{j}}\left(J, \rho_{j}\right)} ; j=1,2
$$

Here $M$ is an operator that may be not linear; $C_{3}$ is a positive constant; the operators $D, M$ and $R_{1}$ are bounded in spaces $L_{q_{j}}\left(J, \rho_{j}\right), \quad j=1,2$.

Then

$$
\left\|D_{1} R_{1}\right\|_{H_{\mu}^{0}(J, \rho)} \leq C_{4} \sum_{j=1}^{2}\left\|D_{1}\right\|_{L_{q_{j}}\left(J, \rho_{j}\right)} \cdot\left\|R_{1}\right\|_{L_{q_{j}}\left(J, \rho_{j}\right)}
$$

where

$$
C_{4}=3 C_{2} C_{3} \max _{j=1,2}\left(\|M\|_{L_{q_{j}}\left(J, \rho_{j}\right)}, 1\right)
$$

We consider the operators

$$
\begin{gathered}
\left(Q_{J} \varphi\right)(x)=\frac{1}{2 x-1} \varphi\left[\frac{x}{2 x-1}\right], \\
\left(S_{J} \varphi\right)(x)=\frac{1}{\pi i} \int_{0}^{1} \frac{\varphi(t) \mathrm{d} t}{x-t} \\
\left(Q_{J} S_{J} \varphi\right)(x)=\frac{1}{\pi i} \int_{0}^{1} \frac{\varphi(t) \mathrm{d} t}{2 x t-x-t}
\end{gathered}
$$

and

$$
\left(V_{k} \varphi\right)(x)=\frac{1}{\pi i} \int_{0}^{1} \frac{\varphi(t) \mathrm{d} t}{t+k x}, \quad k>0
$$

We note that for operators $Q_{J} S_{J}$ and $V_{k}$ conditions (1), (2), (4) of corollary 1 are fulfilled.

Moreover, the following estimations hold

$$
\begin{aligned}
\left|\left(D^{n} Q_{J} S_{J} \varphi\right)(t)\right| & =\mid \frac{1}{\pi i} \int_{0}^{1} \int_{0}^{[}\left[\frac{x(1-x)(1-2 t)}{(x+t-2 t x)^{2}}(1-2 x)^{n-1}-\left(2 \cdot \frac{x^{2}(1-x)^{2}(1-2 t)^{2}}{(x+t-2 t x)^{3}}\right)(n-1)\right] \varphi(t) \mathrm{d} t \\
& \leq C_{5} \cdot \frac{1}{\pi} \int_{0}^{1}\left[\frac{|\varphi(t) \mathrm{d} t|}{x+t-2 t x}\right]=C_{5} \cdot\left|Q_{J} S_{J}\right| \varphi \| ; \quad n=1,2 .
\end{aligned}
$$

where 


$$
C_{5}=\max _{n=1,2} \sup _{x, t \in J}\left|\frac{x(1-x)(1-2 t)}{x+t-2 t x}(1-2 x)^{n-1}-\left(2 \cdot \frac{x^{2}(1-x)^{2}(1-2 t)^{2}}{(x+t-2 t x)^{2}}\right)(n-1)\right|,
$$

and

$$
\begin{gathered}
\left|\left(D^{n} V_{k} \varphi\right)(t)\right|=\left|\frac{1}{\pi i} \int_{0}^{1}\left[\frac{-k x(1-x)}{(t+k x)^{2}}(1-2 x)^{n-1}+\left(2 \cdot \frac{k^{2} x^{2}(1-x)^{2}}{(t+k x)^{3}}\right)(n-1)\right] \varphi(t) \mathrm{d} t\right| \leq C_{6} \frac{1}{\pi} \int_{0}^{1} \frac{|\varphi(t)| \mathrm{d} t}{t+k x}=C_{6} \cdot\left|V_{k}\right| \varphi|| \\
n=1,2,
\end{gathered}
$$

where

$$
C_{6}=\max _{n=1,2} \sup _{x, t \in J}\left|\frac{k x(1-x)}{t+k x}(1-2 x)^{n-1}+\left(2 \cdot \frac{k^{2} x^{2}(1-x)^{2}}{(t+k x)^{2}}\right)(n-1)\right| .
$$

Theorem 2. Let an operator $R_{1}$ be bounded in the space

$$
L_{q_{j}}\left(J, \rho_{j}\right), \quad 1<q_{j}<\infty ; \quad \rho_{j}(x)=x^{r_{j}}(1-x)^{s_{j}} ; \quad-1<r_{j}, \quad s_{j}<q_{j}-1 ; j=1,2,
$$

and inequalities (2) be true.

If

$$
r+\mu>\frac{1+r_{1}}{q_{1}} \text { and } \quad s+\mu>\frac{1+s_{2}}{q_{2}},
$$

then the operators $Q_{J} S_{J} R_{1}$ and $V_{k} R_{1}$ are bounded in space $H_{\mu}^{0}(J, h)$.

Proof. Let a function $\varphi(t)$ belong to $H_{\mu}^{0}(J, \rho)$.

We introduce functions

$$
\varphi_{1}(t)=\chi_{x}(t) \varphi(t), x \in J
$$

and

$$
\varphi_{2}(t)=\varphi(t)-\varphi_{1}(t)
$$

From the fact that

$$
\left(R_{1} \varphi_{j}\right)(t) \in L_{q_{j}}\left(J, \rho_{j}\right), \quad j=1,2
$$

It follows that the function

$$
\Lambda(t)=\frac{\left|\left(R_{1} \varphi_{j}\right)(t)\right|}{t^{1-\frac{1}{2} \lambda_{j}} \cdot(1-t)^{1-\frac{1}{2} \lambda_{j}}}
$$

is summable on segment $J$ if

$$
\frac{1+r_{j}}{q_{j}}+1-\frac{1}{2} \lambda_{j}<1
$$

and

$$
\frac{1+s_{j}}{q_{j}}+1-\frac{1}{2} \gamma_{j}<1 ; \quad j=1,2 .
$$

Condition (6) of the theorem makes it possible to choose constants $\lambda_{j}$ and $\gamma_{j}$ from interval $(0,2)$ so that 


$$
\frac{1+r_{j}}{q_{j}}<\frac{1}{2} \lambda_{j}<r+\mu ; \quad \frac{1+s_{j}}{q_{j}}<\frac{1}{2} \gamma_{j}<s+\mu .
$$

Now, we carry out an estimation of the expression $\left|\rho\left(D^{n} Q_{J} S_{J} R_{1} \varphi\right)\right|$.

In doing so, we will use inequalities (5),

$$
\left|\rho\left(D^{n} Q_{J} S_{J} R_{1} \varphi\right)\right| \leq C_{5} \rho(t)\left|\sum_{j=1}^{2} \frac{1}{\pi i} \int_{0}^{1} \frac{\left(R_{1} \varphi_{j}\right)(\tau) \mid \mathrm{d} \tau}{\tau+t-2 \tau \tau}\right| \leq C_{1} \rho(t)\left|\sum_{j=1}^{2} \frac{1}{\pi i} \int_{0}^{1} \frac{\left|\left(R_{1} \varphi_{j}\right)(\tau)\right| \mathrm{d} \tau}{t^{\frac{1}{2} \cdot \lambda_{j}} \cdot(1-t)^{\frac{1}{2} \cdot \lambda_{j}} \cdot \tau^{1-\frac{1}{2} \cdot \lambda_{j}} \cdot(1-\tau)^{1-\frac{1}{2} \cdot \lambda_{j}}}\right|
$$

where

$$
C_{1}=C_{5} \cdot \sup _{\substack{\tau, t \in J \\ \tau \neq t}}\left|\frac{(t+\tau)(t+\tau-2)}{(t+\tau-2 t \tau)}\right|
$$

Here we have taken into account that

$$
C_{5} \cdot \frac{1}{(t+\tau-2 t \tau)} \leq \frac{C_{1}}{t^{\frac{1}{2} \cdot \lambda_{j}} \cdot \tau^{-\frac{1}{2} \lambda_{j}+1} \cdot(1-t)^{\frac{1}{2} \cdot \lambda_{j}} \cdot(1-\tau)^{1-\frac{1}{2} \lambda_{j}}} .
$$

Since

$$
\rho(t) \cdot t^{-\frac{1}{2} \cdot \lambda_{j}} \cdot(1-t)^{-\frac{1}{2} \cdot \lambda_{j}}=t^{r+\mu-\frac{1}{2} \cdot \lambda_{j}} \cdot(1-t)^{s+\mu-\frac{1}{2} \cdot \gamma_{j}} \rightarrow 0
$$

when $t \rightarrow 0$; and since function $\Lambda(t)$ is summable, it follows that conditions (1) of Theorem 1 are fulfilled for the operator $R=Q_{J} S_{J} R_{1}$.

From properties (5), condition (4) follows:

$$
\left\|D^{n} Q_{J} S_{J} R_{1}\right\|_{L_{q_{j}}\left(J, \rho_{j}\right)} \leq C_{5}\left\|Q_{J} S_{J} M R_{1}\right\|_{L_{q_{j}}\left(J, \rho_{j}\right)},
$$

where $(M \varphi)(X)=|\varphi(x)|$, and as the operator $Q_{J} S_{J}$ is bounded in $L_{q_{j}}\left(J, \rho_{j}\right)$ it follows that all conditions of Corollary 1 are fulfilled and we can apply it. Therefore operator $Q_{J} S_{J} R_{1}$ is bounded in $H_{\mu}^{0}(J, \rho)$.

Since operator $V_{k}$ is bounded in $L_{q_{j}}\left(J, \rho_{j}\right)$, the boundness of operator $V_{k} R_{1}$ in $H_{\mu}^{0}(J, \rho)$ may be proved analogously.

\section{References}

[1] Gakhov, F.D. (1977) Boundary Value Problems. Nauka, Moscow. (in Russian)

[2] Muskhelishvili, N.I. (2008) Singular Integral Equations, Boundary Value Problems of the Theory of Functions and Some of Their Applications to Mathematical Physics. Dover Publications, Mineola.

[3] Litvinchuk, G.S. (2000) Solvability Theory of Boundary Value Problems and Singular Integral Equations with Shift. Kluwer Academic Publishers, Dordrecht, Boston, London. http://dx.doi.org/10.1007/978-94-011-4363-9

[4] Gohberg, I. and Krupnik, N. (1992) One-Dimensional Linear Singular Integral Equations. Operator Theory: Advances and Applications Vol. 53. Birkhauser Verlag, Basel, Boston, Berlin.

[5] Mikhlin, S.G. and Prossdorf, S. (1986) Singular Integral Operators. Akademie-Verlag, Berlin. http://dx.doi.org/10.1007/978-3-642-61631-0

[6] Duduchava, R.V. (1963) Unidimensional Singular Integral Operator Algebras in Spaces of Holder Functions with Weight. Proceedings of A. Razmadze Mathematical Institute, 43, 19-52. (in Russian)

[7] Karapetiants, N.K. and Samko, S.G. (2001) Equations with Involutive Operator. Birkhauser Verlag, Boston, Basel, Berlin. http://dx.doi.org/10.1007/978-1-4612-0183-0

[8] Duduchava, R.V. (1979) Convolution Integral Equations with Discontinuous Presymbols, Singular Integral Equations with Fixed Singularities and Their Applications to Problem in Mechanics. Proceedings of A. Razmadze Mathematical 
Institute, 60, 2-136. (in Russian)

[9] Karlovich, Yu. and Kravchenko, V. (1981) Singular Integral Equations with Non-Carleman Shift on an Open Contour. Differential Equations, 17, 1408-1417.

[10] Kravchenko, V.G. and Litvinchuk, G.S. (1994) Introduction to the Theory of Singular Integral Operators with Shift. Kluwer Academic Publishers, Dordrecht, Boston, London. http://dx.doi.org/10.1007/978-94-011-1180-5

[11] Karelin, A. (1980) On a Boundary Value Problem with Shift for a System of Differential Equations of Elliptichyperbolic Type. Soviet Mathematics-Doklady, 22, 507-512. 
Scientific Research Publishing (SCIRP) is one of the largest Open Access journal publishers. It is currently publishing more than 200 open access, online, peer-reviewed journals covering a wide range of academic disciplines. SCIRP serves the worldwide academic communities and contributes to the progress and application of science with its publication.

Other selected journals from SCIRP are listed as below. Submit your manuscript to us via either submit@scirp.org or Online Submission Portal.
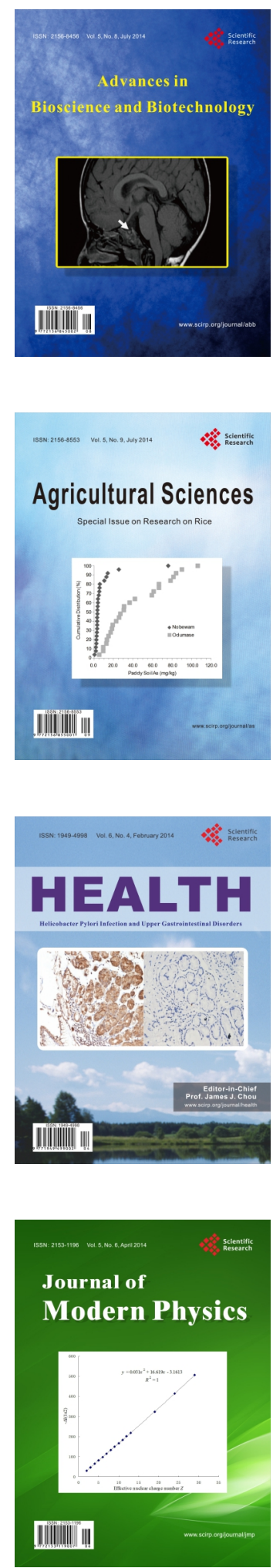
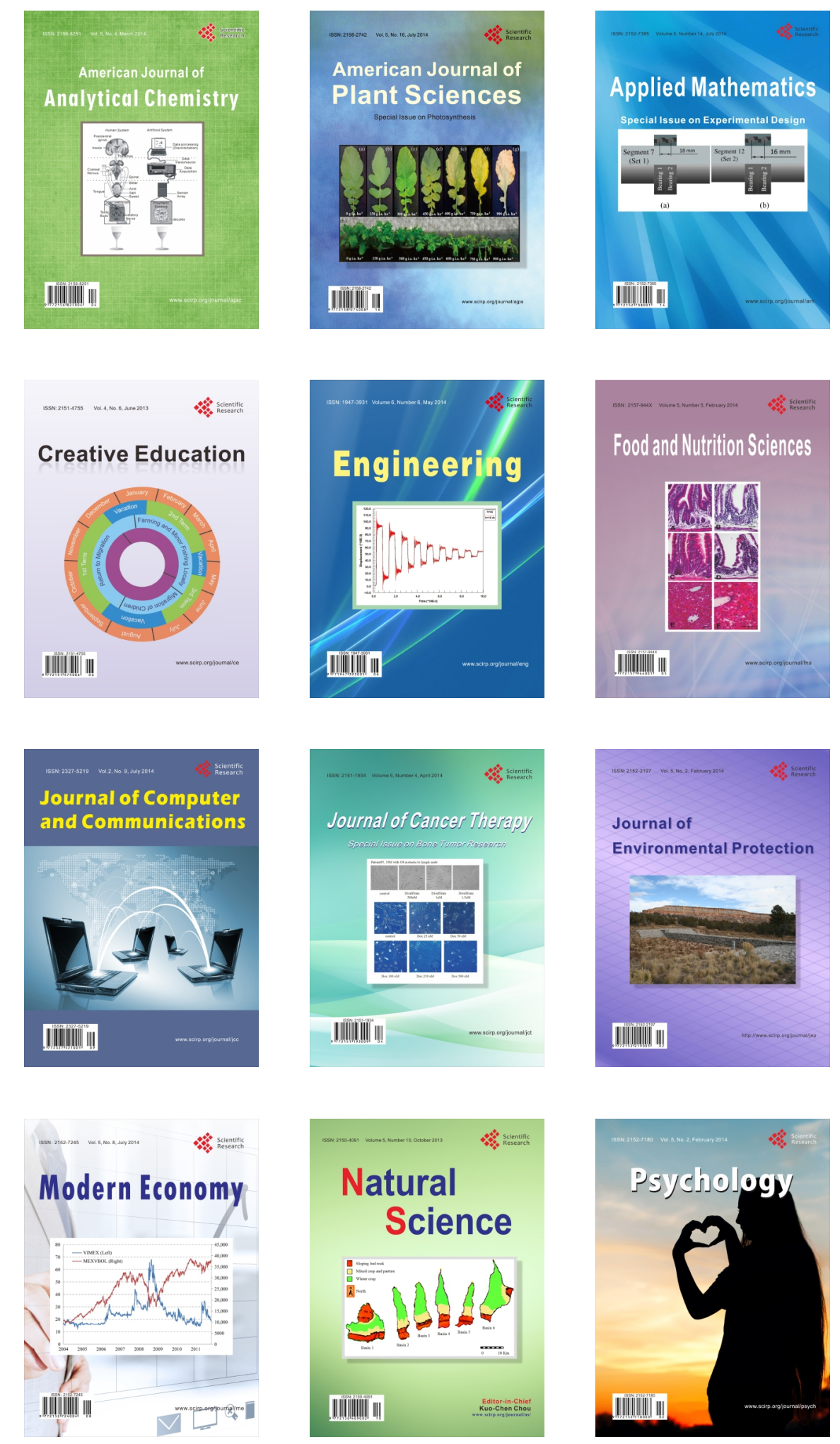